\title{
Access to Preventive Health Services of People with Intellectual Disability in Italy
}

\author{
Aldo Rosano ${ }^{1, *}$ and Alessandra Battisti ${ }^{2}$ \\ ${ }^{1}$ National Institute for Public Policies Analysis, Rome, Italy \\ ${ }^{2}$ National Institute of Statistics, Rome, Italy
}

\begin{abstract}
People with intellectual disabilities today have an high life expectancy, routine screening and preventive services are essential to their overall quality of care. We used the 2012-2013 Italian National Health Interview Survey (NHIS) to examine screening and preventive service use for persons with intellectual disability. We estimated about 953 thousand people with intellectual disability, $58 \%$ of which are female and the $59 \%$ are over 65 years. Their quality of life, in terms of physical and mental status, was significant lower than general population. Intellectually disabled women generally reported screening and preventive services at lower as compared to women without intellectual disability: the risk of not attending a Pap smear test in a due time, in terms of odds ratio, was 4.5 (95\% confidence interval, $3.6-5.7)$, as for mammography was 2.4 (95\% confidence interval, 1.8-3.2). The risk of not attending colorectal screening was also significant, even if lower than female screening, (OR: 1.5; 95\% confidence interval, 1.2-2.0). Various approaches can be adopted to improve access for intellectually disabled persons to preventive health care services.
\end{abstract}

Keywords: Intellectual disability, preventive health services, screening, mental health, access.

\section{INTRODUCTION}

When compared to the general population, persons with an intellectual disability (ID) have lower life expectancy, higher morbidity, and more difficulty finding and obtaining healthcare. Adults with an ID often have difficulty in receiving the healthcare they need. Compared to other persons who do not have an ID, they have poorer health and have more difficulty finding, getting to, and paying for healthcare. This happens for both physical and mental healthcare needs [1].

People with ID might be unaware of physical problems and might have difficulty in verbally expressing such conditions, for this reason they usually access the healthcare services only when their conditions become severe.

People with ID are less likely to receive preventive healthcare [2-4]. For instance, only $25 \%$ of women with ID in England underwent cervical screening [5] and remained low even after a supportive intervention aimed at increasing uptake. Conversely, breast screening in women with moderate to severe ID appeared to be better accessed and not so different from general population [6]. As well as coverage of cervical cancer screening in women with ID is markedly lower than for the general population [7].

*Address correspondence to this author at the National Institute for Public Policies Analysis, Corso d'Italia 33, Rome, Italy; Tel: +39-06-49903551; Fax: +39-06-85447358; E-mail: a.rosano@inapp.org
The available evidence shows as people with disabilities encounter disparities in many fields. They experienced a history of social, economic, and environmental disadvantages in which children and adults with disabilities were institutionalized and marginalized. There are differences in health outcomes that relate to higher rates of unmet health care needs, unhealthy bad lifestyle behaviours, poor mental health and chronic diseases, and deprivation. Many of these differences disproportionately affect this population and can be avoided through targeted policies [8]. People with ID experience inequities in health status and access to health systems as well. Reliable information is very important to identify and quantify such health disparities.

A recent review of health interview and health examination surveys conducted in 17 European countries revealed that only few surveys were identified as having potential to extract data on respondents with ID [9].

The objective of the study is to compare the mental and physical status and the access to preventive health services (PHS) between people with intellectual disabilities (ID) and the rest of the population (ROP), while considering some factors that may confound or interact with the relationship between access to PHSs, in Italy.

\section{METHODS} study.
Study design: retrospective period prevalence 
Data Sources: Data from the Italian Health Interview Survey (HIS) 2012-13, conducted by the National Institute of Statistics (ISTAT), with 119,007 interviewed subjects.

People with ID were detected within the survey through the following question relevant to the POMOMA 18 indicator set [9]: "Are you affected by one of the following invalidity? Mental retardation or mental disease and behavioural disorders".

We analysed individual information from HISs regarding the Physical and Mental Health Composite Scale scores (PCS \& MCS), the access to mammography, Pap smear tests, and colorectal cancer screening. Additionally, we analysed data on factors potentially influencing the relationship between access to PHSs and status of ID, such as age, sex and geographical areas.

PCS and MCS were computed using the scores of twelve questions (SF-12). Each indicator range from 0 to 100 , where a zero score indicates the lowest level of health measured by the scales and 100 indicates the highest level of health.

We also considered three indicators regarding PHS access, according to the European Core Health Indicators' definitions [10]:

Percentage of women (aged 50-69) reporting a mammography in the past 2 years

Percentage of women (aged 20-69) reporting a Pap smear test in the past 3 years

Percentage of persons (aged 50-74) reporting a colorectal cancer screening in the past 2 years
Statistical methods: a descriptive analysis was conducted calculating the percentage of the selected indicators by the presence of ID. Mean values of PCS and MCS were compared between persons with or without ID by age class using a t-test. Finally, in order to compare the risk of persons with ID of not accessing PHS with that of the rest of populations (ROP), odds ratios (ORs) were estimated using a logistic regression models adjusted for potential confounders, using ROP as a reference.

\section{RESULTS}

The estimate number of people with ID in Italy amount to 953 thousand. The $58 \%$ are female and the $59 \%$ are over 65 years old. The age structure of the population with ID are different between the geographical areas. In the Centre there are much more elderly than in the North and in the South areas.

\section{Physical and Mental Health}

People with ID have lower value of the Physical and Mental Health Composite Scale than the rest of population. This result underlines the vulnerability of the health and psychological status of this population (Table 1).

\section{Female Screening}

Cancer screening analysis seems to be less accessible to women with ID than women without ID. The geographical differences, related to Pap smear test, between the two population aged 25-69 years cover a range between the $19 \%$ in the South of Italy and the $41 \%$ in the Centre. However, differences in

Table 1: Physical and Mental Health Composite Scale mean scores in people aged 14+ by presence of intellectual disability and age class and p-values of the t-tests. Italy, 2013

\begin{tabular}{|c|c|c|c|c|c|c|}
\hline \multirow{2}{*}{ Age classes } & \multicolumn{2}{|c|}{ Persons without intellectual disability } & \multicolumn{2}{|c|}{ Persons with intellectual disability } & \multirow{2}{*}{$\begin{array}{c}\text { t-test PCS } \\
\text { p-value }\end{array}$} & \multirow{2}{*}{$\begin{array}{c}\text { t-test MCS } \\
p \text {-value }\end{array}$} \\
\hline & PCS & MCS & PCS & MCS & & \\
\hline $14-24$ & 55.8 & 51.6 & 44.0 & 42.7 & $p<0.01$ & $p<0.01$ \\
\hline $25-34$ & 54.8 & 50.5 & 44.5 & 39.5 & $p<0.01$ & $p<0.01$ \\
\hline $35-44$ & 54.0 & 50.0 & 42.6 & 36.4 & $p<0.01$ & $p<0.01$ \\
\hline $45-54$ & 52.5 & 48.7 & 40.3 & 34.9 & $p<0.01$ & $p<0.01$ \\
\hline $55-64$ & 50.2 & 48.6 & 36.6 & 33.6 & $p<0.01$ & $p<0.01$ \\
\hline $65-69$ & 47.9 & 48.9 & 34.6 & 34.1 & $p<0.01$ & $p<0.01$ \\
\hline $70-74$ & 45.6 & 47.9 & 30.8 & 32.9 & $p<0.01$ & $p<0.01$ \\
\hline $75-79$ & 42.5 & 46.8 & 28.7 & 35.0 & $p<0.01$ & $p<0.01$ \\
\hline $80+$ & 37.4 & 45.9 & 27.1 & 34.3 & $p<0.01$ & $p<0.01$ \\
\hline Total & 51.0 & 49.2 & 33.6 & 35.1 & $p<0.01$ & $p<0.01$ \\
\hline
\end{tabular}


Table 2: Percentage of women aged 25-64 year who have attended a Pap smear test in the past 3 year or a mammography in the last 2 years, by presence of intellectual disability and area of residence. Italy, 2013

\begin{tabular}{|c|c|c|c|c|}
\hline & \multicolumn{2}{|c|}{ Pap smear test } & \multicolumn{2}{c|}{ Mammography } \\
\hline Geographical areas & $\begin{array}{c}\text { Persons without } \\
\text { intellectual disability }\end{array}$ & $\begin{array}{c}\text { Persons with intellectual } \\
\text { disability }\end{array}$ & $\begin{array}{c}\text { Persons without } \\
\text { intellectual disability }\end{array}$ & $\begin{array}{c}\text { Persons with intellectual } \\
\text { disability }\end{array}$ \\
\hline \hline North & 79.6 & 49.7 & 86.0 & 70.8 \\
\hline Centre & 75.7 & 34.8 & 80.3 & 66.0 \\
\hline South & 46.9 & 28.3 & 55.7 & 40.3 \\
\hline Italy & $\mathbf{6 7 . 5}$ & $\mathbf{3 7 . 4}$ & $\mathbf{7 4 . 7}$ & $\mathbf{5 4 . 2}$ \\
\hline
\end{tabular}

Table 3: Percentage of persons aged 50-74 year who have attended a colorectal screening test in the past 2 year by presence of intellectual disability, sex and area of residence. Italy, 2013

\begin{tabular}{|c|c|c|c|c|c|c|}
\hline \multirow{2}{*}{$\begin{array}{c}\text { Geographical } \\
\text { areas }\end{array}$} & \multicolumn{2}{|c|}{ Persons without intellectual disability } & \multicolumn{3}{c|}{ Persons with intellectual disability } \\
\cline { 2 - 7 } & Males & Females & Total & Males & Females & Total \\
\hline \hline North & 54.2 & 52.9 & 53.5 & 25.4 & 37.9 & 31.8 \\
\hline Centre & 39.2 & 37.5 & 38.3 & 36.7 & 17.2 & 16.6 \\
\hline South & 15.9 & 13.1 & 14.5 & $\mathbf{2 3 . 0}$ & $\mathbf{2 6 . 6}$ & $\mathbf{2 4 . 8}$ \\
\hline Italy & $\mathbf{3 8 . 5}$ & $\mathbf{3 6 . 4}$ & $\mathbf{3 7 . 4}$ & & & 36.7 \\
\hline
\end{tabular}

having a mammography among women with ID is apparent in all the geographical areas (Tables 2, 3). The recourse to these two cancer screening are very low in women with ID. The risk of not attending a mammography in a due time was more than double in women with ID (OR=2.4; 95\% confidence interval, 1.83.2) when compared to women without ID, and even higher for Pap smear test (OR=4.5; 95\% confidence interval, 3.6-5.7). The propensity to attend the test is lower in Southern regions of Italy and increases, especially for the Pap smear test, with age (data not shown).

\section{Colorectal Screening}

The differences in access to colorectal cancer screening are higher in the areas where the coverage of screening protocol is widespread, in the geographical areas where the coverage is lower the differences between population with and without disability are lower as well (North vs South). The logistic regression model point out the same differences: the risk of not attending a colorectal screening in a due time was significantly higher in subjects with ID (OR: 1.5; 95\% confidence interval, 1.22.0) when compared to those without ID. The propensity decrease from North to South and have a significant increase in the population aged 60-74 (OR: $1.5 ; 95 \%$ confidence interval, 1.4-1.6) (data not shown).

\section{DISCUSSION}

The fragility of population with ID should imply a privileged access PHS. However, the analysis of the access for these services put in evidence a significant gap in the receiving PHS when compared to persons without ID, especially in the areas with lower level of provision of such health services.

Health prevention programmes and programs for positive health behaviour as well as access to information and programs to avoid health risk behaviour for people with ID is lacking. This aspect of health is central in order to strengthen the empowerment of young people and adults with intellectual disabilities [11].

Persons with ID experience obstacles to accessing high-quality healthcare, and this implies higher cost of healthcare. People with ID access have a limited access to health promotion resources and preventative healthcare through primary care. Consequently, health conditions are left unaddressed until they require far more extensive and expensive interventions [12]. We showed as the risk of not accessing screening for people with ID is more than doubled in comparison with people without ID.

It is difficult for a person with ID to face an invasive analysis with unknown staff. There is evidence that 
access to vaccination against flu that was distributed by GPs is easier than access to cancer screening distributed by hospital and private doctor. Performing even simple diagnostic and therapeutic performance may be very complex [13]. The difficulties of having a relationship with the patient make it a reality that in many patients with ID numerous medical problems are neglected, especially in the prevention programs.

Moreover, access to the prevention of people with intellectual disabilities obviously be influenced by the territorial differences of access of the whole population to these types of services. In our study we observed as the differences are greater when the coverage of the whole population on the single area is greater, where the covering is very low for the whole population, the differences between the two populations, with and without ID, decrease. These results underline that a lower differences is not a measure of a minor discrimination but it could be a greater inefficiency of the territorial area to apply the prevention programs.

There are different strategies to improve the access to health services of persons with ID, such as increasing the intensity and frequency of service delivery or improving the training of health care providers about adults ID. However, there is scarce evidence on different ways to organise services for people with ID [1] and, in general, health professionals are poorly prepared to meet the complex medical and psychosocial needs of people with ID [8]. Possible actions include strengthening health services workforce capacity in dealing with persons with ID and involving the associations for persons with ID in the organization of public health programs.

\section{REFERENCES}

[1] Balogh R, Ouellette-Kuntz $H$, Bourne L, Lunsky $\mathrm{Y}$, Colantonio A. Organising health care services for persons with an intellectual disability. Cochrane Database Syst Rev 2008; 4.

https://doi.org/10.1002/14651858.CD007492
[2] Whitfield $M$, Langan $J$, Russell $O$. Assessing general practitioners' care of adult patients with learning disability: case-control study. Quality and Safety in Health Care 1996; $5(1): 31-5$.

https://doi.org/10.1136/qshc.5.1.31

[3] Lennox NG, Diggens J, Ugoni A. Health care for people with an intellectual disability: general practitioners' attitudes, and provision of care. Journal of Intellectual and Developmental Disability 2000; 25(2): 127-33. https://doi.org/10.1080/13269780050033544

[4] Tezzoni LI, McCarthy EP, Davis RB, Harris-David L, O'day B. Use of screening and preventive services among women with disabilities. American Journal of Medical Quality 2001; 16(4): 135-44. https://doi.org/10.1177/106286060101600405

[5] Pearson V, Davis C, Ruoff C, Dyer J. Only one quarter of women with learning disability in Exeter have cervical screening. BMJ 1998; 316(7149): 1979.

https://doi.org/10.1136/bmj.316.7149.1979

[6] Biswas M, Whalley H, Foster J, Friedman E, Deacon R. Women with learning disability and uptake of screening: audit of screening uptake before and after one to one counselling. Journal of Public Health 2005; 27(4): 344-7.

https://doi.org/10.1093/pubmed/fdi055

[7] Stein K, Allen N. Cross sectional survey of cervical cancer screening in women with learning disability. Bmj. 1999; 318(7184): 641.

https://doi.org/10.1136/bmj.318.7184.641

[8] Krahn GL, Walker DK, Correa-De-Araujo R. Persons with disabilities as an unrecognized health disparity population. American Journal of Public Health 2015; 105(S2): S198S206.

https://doi.org/10.2105/AJPH.2014.302182

[9] Linehan C, Walsh P, Van Schrojenstein Lantman-de Valk $\mathrm{H}$ Kerr MP, Dawson F. Are people with intellectual disabilities represented in European public health surveys? Journal of Applied Research in Intellectual Disabilities 2009; 22(5): 40920

https://doi.org/10.1111/j.1468-3148.2009.00521.x

[10] Fehr A, Lange C, Fuchs J, Neuhauser H, Schmitz R. Health monitoring and health indicators in Europe 2017.

[11] Inclusion Europe. Inclusive health and people with intellectual disabilities - Position Paper of Inclusion Europe Bruxelles 2012.

[12] Ervin DA, Merrick J. Intellectual and developmental disability: healthcare financing. Frontiers in Public Health 2014; 2.

[13] Alborz A, McNally R, Glendinning C. Access to healthcare for people with learning disabilities: Mapping the issues and reviewing the evidence. J Health Serv Res Policy 2005; 10: 173-182.

https://doi.org/10.1258/1355819054338997

\section{DOI: https://doi.org/10.6000/2292-2598.2017.05.03.5}

(C) 2017 Rosano and Battisti; Licensee Lifescience Global.

This is an open access article licensed under the terms of the Creative Commons Attribution Non-Commercial License (http://creativecommons.org/licenses/by-nc/3.0/) which permits unrestricted, non-commercial use, distribution and reproduction in any medium, provided the work is properly cited. 